\title{
VALIDATION OF DELAYED GADOLINIUM-ENHANCED MAGNETIC RESONANCE IMAGING OF CARTILAGE AND T2 MAPPING FOR QUANTIFYING DISTAL METACARPUS/METATARSUS CARTILAGE THICKNESS IN THOROUGHBRED RACEHORSES
}

\author{
ANN CARSTENS, ROBERT M. KIRBERGER, , LEIF E. DAHLBERG, \\ LEON PROZESKY, LIZELLE FLETCHER, EVELIINA \\ LAMMENTAUSTA
}

From the Section of Diagnostic Imaging, Department of Companion Animal Clinical Studies (Carstens, Kirberger), and the Department of Pathology (Prozesky), Faculty of Veterinary Science, Private Bag X04 Onderstepoort 0110; and the Department of Statistics (Fletcher), University of Pretoria, South Africa, and the Oulu University Hospital, Department of Diagnostic Radiology, POB 50, FI-90029 OYS, Oulu, Finland (Lammentausta); and the Joint and Soft Tissue Unit, Department of Clinical Sciences, Malmö, Lund University, Department of Orthopaedics, Malmö University Hospital, SE-205 02 Malmö, Sweden (Lammentausta, Dahlberg)

Supported by the University of Pretoria Veterinary Faculty Research Fund, the Department of Companion Animal Clinical Studies and a grant to Prof RM Kirberger from the National Research Foundation of South Africa.

Address correspondence to: Ann Carstens, Section of Diagnostic Imaging, Department of Companion Animal Clinical Studies, Faculty of Veterinary Science, University of Pretoria, Private Bag X04, ONDERSTEPOORT, 0110, Republic of South Africa. E-mail: ann.carstens@up.ac.za Tel.: +27-125298012; Fax.: +27-12-5298307.

Acknowledgments: The authors would like to thank Mrs J Jordaan and Dr L Fletcher, of the Department of Statistics, University of Pretoria, South Africa for their assistance with statistical analyses. 


\section{Abstract}

The purpose of this study was to determine whether Delayed Gadolinium-enhanced Magnetic Resonance Imaging of Cartilage (dGEMRIC) and T2 mapping are accurate techniques for measuring cartilage thickness in the metacarpus3/metatarsus3 (Mc3/Mt3) of Thoroughbred racehorses. Twenty-four Mc3/Mt3 cadaver specimens were acquired from 6 healthy racehorses. Cartilage thickness was measured from post- intra-articular Gd-DTPA ${ }^{2-}$ images acquired using short tau inversion recovery (STIR), and proton density weighted (PDw) sequences, and compared with cartilage thickness measured from corresponding histologic images. Two observers performed each histologic measurement twice at 3 different sites, with measurement times spaced at least 5 days apart. Histologic cartilage thickness was measured at each of the 3 sites from the articular surface to the bone-cartilage interface, and from the articular surface to the mineralised cartilage interface (tidemark). Intra-observer repeatability was good to moderate for dGEMRIC where $\mathrm{Mc} 3 / \mathrm{Mt} 3$ cartilage was not in contact with the proximal phalanx. Where the Mc3/Mt3 cartilage was in contact with the proximal phalanx cartilage, dGEMRIC STIR and T2 mapping PDw cartilage thicknesses of Mc3/Mt3 could not be measured reliably. When measured from the articular surface to the bone-cartilage interface, histologic cartilage thickness did not differ from STIR or PDw cartilage thickness at the site where the Mc3/Mt3 cartilage surface was separated from the proximal phalanx cartilage $(p>0.05)$. Findings indicated that dGEMRIC STIR and T2 mapping PDw are accurate techniques for measuring Mc3/Mt3 cartilage thickness at locations where the cartilage is not in direct contact with proximal phalanx cartilage. 


\section{Introduction}

Lameness is the primary cause of poor performance and wastage in horses. ${ }^{1-6}$ This decrease in athletic ability has been estimated to cost the performance horse industry in North America an estimated $\$ 1$ billion annually, with an incidence of $8.5 \%$ to $13.7 \%{ }^{7}$ In studies performed at racetracks throughout the world, the most common source of lameness is the distal limb. ${ }^{5,8-13}$ Twenty five percent of racing Thoroughbreds experience metacarpophalangeal and metatarsophalangeal joint pain, ${ }^{5}$ with this joint being the most commonly affected by traumatic and degenerative lesions of the appendicular skeleton resulting in osteoarthritis. ${ }^{6,10,14,15,16}$ Osteoarthritis is characterized by matrix fibrillation, the appearance of fissures and ulceration and full-thickness loss of the cartilage. ${ }^{17-20}$

Magnetic resonance imaging, including the use of gadolinium $\left(\mathrm{Gd}-\mathrm{DTPA}^{2-}\right)$ has been used in human studies to visualize osteoarthritis early in the disease process. ${ }^{21-25}$ Parametric mapping of cartilage entails post-processing of images to give relaxation time-associated colour-maps that provide a visual interpretation of the specific area's relaxation times. This has been described in $\mathrm{T} 2, \mathrm{~T} 2 *$, and delayed gadolinium enhancement of MR in cartilage (dGEMRIC)) techniques. ${ }^{26} \mathrm{~T} 2$-mapping is a non-invasive technique that can characterize hyaline articular cartilage and repair tissue. $^{27}$ In dGEMRIC, the negatively charged Gd-DTPA ${ }^{2-}$, injected either intraarticularly or intravenously, penetrates hyaline cartilage in an inverse relationship to the proteoglycan concentration of the cartilage. When proteoglycan concentration is decreased due to cartilage degradation, as seen in osteoarthritis, the penetration of Gd$\mathrm{DTPA}^{2-}$ is increased due to a relative decrease in negative charge of the proteoglycandepleted cartilage. Delayed gadolinium enhanced MR in cartilage has been shown to be an excellent indicator of early degenerative cartilaginous changes in humans. ${ }^{24}$ The 
MRI parameter maps for T2 and dGEMRIC can be made by freehand drawing of cartilage ROIs. Signal intensity can be then fitted pixel by pixel into monoexponential relaxation equations using image analysis software (MATLAB Mathworks Inc., Natick, MA, USA). Results of these calculations yield a relaxation time value for each pixel. A mean of the relaxation time values for pixels within an ROI can be then used to characterize the cartilage within each region of interest (ROI).

To ensure that the bone/cartilage interface and the cartilage surface of the dGEMRIC and T2 mapping sites are consistent with true anatomical areas, previous validation studies have compared MRI measurements to histological measurements as the gold standard. A study validating MRI imaging measurements of equine carpal cartilage thickness found a significant correlation between gradient echo and spoiled gradient echo, and spoiled gradient echo and histologic measurements. ${ }^{28}$ The same study found that, when calcified cartilage was excluded from the histologic measurement, MRI measurements were significantly greater than histologic measurements. $^{28}$

Another previous human study reported good repeatability for cartilage thickness measurements using a 7T scanner, with a coefficient of variation of $1.13 \%{ }^{29}$ Good repeatability of MRI cartilage thickness measurements was also found in a study of asymptomatic human hip joints. ${ }^{30}$ Inter-rater and intra-rater reliability of human cadaver femoral head cartilage thickness measurements from 3D- spoiled gradient echo pulse sequences have been found to be very high $(<0.98)^{31}$ and highresolution MRI cartilage thickness evaluation was also found to have good correlation with direct imaging analysis of surgically removed cartilage. ${ }^{32}$ For accurate determination of highly curved and thin articular cartilage volume and thickness 
three-dimensionally, a 3D gradient echo sequence with selective water excitation acquisition can be used together with semi-automatic segmentation using a spline Snake algorithm. ${ }^{33}$

Previous studies have also validated MRI cartilage measurement techniques for assessing progression of osteoarthritis. In one previous study, MRI detected a 1$2 \%$ decrease in cartilage thickness annually in human patients with some identifiable risk factors. ${ }^{24}$ Another study reported that MRI cartilage thickness and volume measurements decrease in patients with symptomatic knee osteoarthritis. ${ }^{34}$ In an experimental study using a guinea pig meniscectomy model, MRI cartilage thickness measurement precision (repeatability) was good, with positive agreement and a significant partial correlation between measurements. ${ }^{35}$ Cartilage thickness changes were also seen in serial MRI examinations of guinea pig stifle joints after meniscectomy. ${ }^{36}$ Computer aided methods for quantifying cartilage thickness and volume changes using MRI have also been validated. ${ }^{37}$ A computer aided technique where measurements were tested and re-tested (paired imaging analysis), improved precision of cartilage segmentation for articular surfaces of the femur, tibia and patella was found. ${ }^{38}$ Using MRI-based 3D cartilage models, the thickness of cartilage was overestimated in regions where cartilage thickness was $<2.5 \mathrm{~mm}$ and correctly predicted in regions where the cartilage was $>2.5 \mathrm{~mm} .^{39}$

In a study comparing a standard MRI knee protocol and T2-mapping, T2mapping was found to be feasible in a clinical setting and also revealed cartilage lesions not visible with standard clinical MRI protocols. ${ }^{40}$ Day-to-day repeatability of the dGEMRIC measurements has also been reported at different knee joint surfaces of healthy humans, and has been found to be good for small, deep or superficial segments, for full thickness ROIs and for bulk ROIs. ${ }^{41}$ 
The purpose of the current study was to validate T2 mapping and dGEMRIC techniques for measuring cartilage thickness in Thoroughbred racehorses, using three selected sites of the distal Mc3/Mt3 condyle of normal Thoroughbred cadaver specimens. The first objective of the study was to determine intra- and inter-observer agreement for histomorphometrical measurements of cartilage thickness for the three sites, measured from the articular surface to the most superficial bone Haversian canals (bone cartilage interface) and to the most superficial aspect of mineralised cartilage (tidemark (mineralized cartilage interface)). The second objective was to determine intra-observer agreement for measurements of cartilage thickness for the three sites and for two MRI techniques, the TI=200ms (hereafter referred to as the STIR) images during dGEMRIC-mapping and the $\mathrm{TE}=16.7 \mathrm{~ms}$ (hereafter referred to as PDw (proton density weighted)) images during T2-mapping. The third objective was to compare histologic measurements of bone cartilage interface and mineralized cartilage interface to cartilage surface with measurements of cartilage thickness from the STIR images in dGEMRIC mapping and the PDw images in T2 mapping. The last objective was to compare cartilage thickness measurements from the STIR images in dGEMRIC mapping to measurements from the PDw images in T2 mapping.

\section{Materials and Methods}

This was a prospective cross-sectional study using 24 cadaver limbs of 6 clinically sound Thoroughbred horses. The project (V067/10) was approved by the Animal Use and Care Committee of the Faculty of Veterinary Science University of Pretoria and all horses were treated according to South African Veterinary Council ethical standards. Inclusion criteria were that the horses were to be Thoroughbreds aged between 3 and 6 years, of any gender, with no pain on flexion of the 
metacarpophalangeal or metatarsophalangeal joints and no signs of lameness at a walk or trot. There had to be no clinically apparent signs of marked appendicular skeletal abnormalities and no history of corticosteroid or glycosaminoglycan treatments in the past week. The horses were obtained from a welfare horse care organisation where racing Thoroughbreds are sent for adoption or euthanasia.

The horses were shot intra-cranially and the limbs removed at the middiaphyses of the third metacarpal or metatarsal bones. The limbs were wrapped in cling plastic, identified, placed in a cool bag and transported to a MRI facility (Pretoria MR Trust, Faerie Glen Branch, Pretoria, South Africa) with a Siemens Avanto 1.5 T MRI machine with A B17 Software upgrade (Siemens Healthcare, Erlangen, Germany). The scanning took place with the limbs at room temperature $\left(20^{\circ} \mathrm{C}\right)$ approximately six hours post euthanasia. A vitamin E oil capsule was taped to the lateral aspect of every Mc3/Mt3 specimen. The limbs were placed on the scanner table with the dorsal surface facing down and with the toe facing into the gantry. A head and neck 12-channel receiver coil combination was used. The forelimbs were randomly selected and scanned, followed by those of the randomly selected hind limbs. A leap-frog time schedule was used to ensure optimal time use, with the four metacarpo-/metatarsophalangeal joints' scans finishing approximately 8 hours after commencement of the scans. The T1-weighted images were acquired using turbo spin echo sequences in the sagittal plane with TR: 557ms; TE: 23ms; FOV: 100x100; matrix: 256x256; and receiver band-width: $130 \mathrm{~Hz} /$ pix. The T2-mapping images were acquired using multi-slice multi-echo spin echo sequences with TR: 2170ms; six TEs at between 16.7 and 116.9ms; FOV: 140x140; matrix: 256x256; slice thickness: 3mm; and $\mathrm{kHz}$ receiver band-width: $130 \mathrm{~Hz} /$ pix. A 17-line software template was used on a 
transverse localizer of the distal Mc3/Mt3 to identify the mid-lateral Mt3 condyle and the mid-medial Mc3 condyle (Figure 1A).
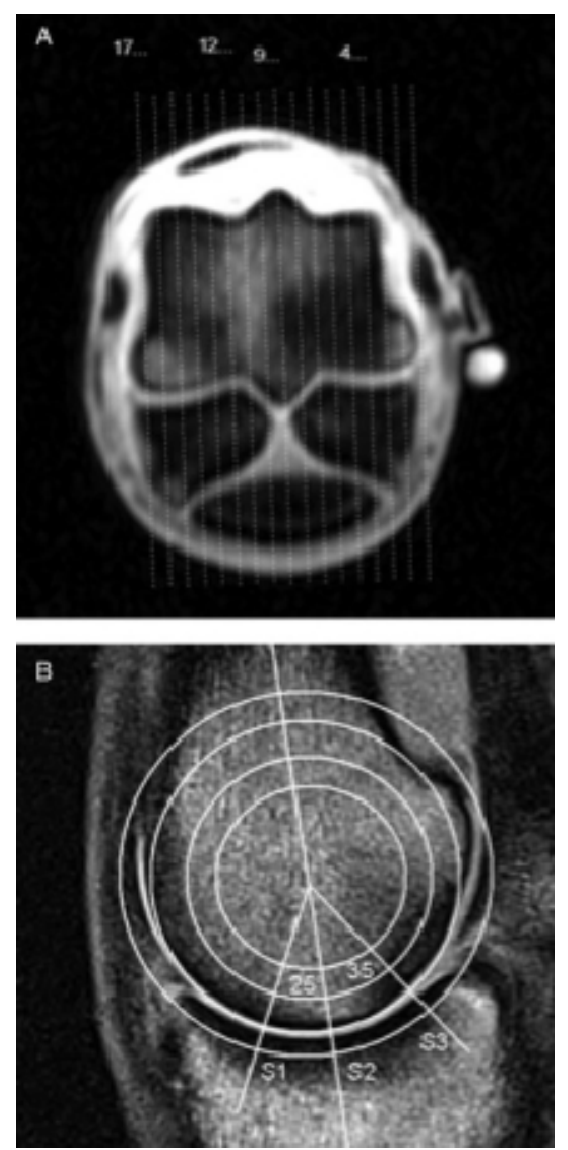

FIG. 1A. Transverse pilot MRI image of the distal third metatarsal bone of a normal Thoroughbred horse, demonstrating how MRI slices were marked for histologic comparisons. A 17-line template has been placed on the slice and centered over the distal sagittal ridge. Slice 1 is positioned on the lateral epicondyle cortex and slice 17 extends slightly beyond the medial epicondyle cortex. A hyperintense vitamin E oil capsule has been used as a marker for the lateral epicondylar surface.

FIG. 1B. Lateral mid-condylar parasagittal PDw (of T2 mapping) MRI image with a representation of the translucent template illustrating concentric circles placed using a "best-of-fit" method over the third metacarpal condyle and with a line transecting the midline of the Mc3 distal metaphysis. Sites 1-3 are also identified.

To acquire the parasagittal images for dGEMRIC T1 relaxation time calculation, 6 pre-contrast inversion recovery sequences were performed on the mid 
lateral condyle for the hind limb and the mid medial condyle for the forelimb, respectively. As much synovial fluid as possible was aspirated from the palmar/plantar recess of the metacarpo-/metatarsophalangeal joints to minimise dilution of the Gd-DTPA ${ }^{2-}$ and minimize fluid-related sources of variability. Gadolinium-DTPA ${ }^{2-}$ (Magnevist ${ }^{\circledR}$, gadopentetate dimeglumine, Bayer Health Care Pharmaceuticals, Isando, Gauteng, South Africa) was injected at $0.05 \mathrm{ml}$ in $5 \mathrm{ml}$ saline $(0.025 \mathrm{mmol} /$ joint $)$. The joints were manually flexed at approximately one flexion per second for 5 minutes to ensure adequate contrast distribution in all parts of the joint. The same 6 inversion recovery T1 sequences as those used for pre-contrast images were repeated at 30,60,120, and 180 minutes post-injection and the same mid-condylar areas. The joint fluid was examined cytologically the following day and the balance aliquoted and frozen at $-80^{\circ}$ for later further analysis. Three distal articulation sites of Mc3/Mt3 were identified for histological and MRI measurements. Site 1 was defined based on a $25^{\circ}$ dorsal angle and from a point in the center of the rotation of the joint. Site 2 was defined as the distal aspect of a line drawn down the axis of the diaphysis of Mc3/Mt3 and corresponding to the transverse ridge. Site 3 was defined based on a $35^{\circ}$ palmar/plantar angle from a point in the center of the rotation of the joint and using the sagittal template of slices 5 and 13 (Fig. 1B). The palmar site location was chosen based on the site where cartilage injury has been previously reported, most often in conjunction with subchondral sclerosis and signs of fatigue condylar fractures. ${ }^{42,43}$ The locations of sites 1-3 were chosen to be the same as sites previously evaluated by researchers studying stages of condylar fatigue fractures $^{42}$.

The metacarpo-/metatarsophalangeal joints were radiographed and scanned with computed tomography and limbs discarded from this study if there were overt 
signs of pathology, such as osteochondritis dissecans or osteoarthritis changes with osteophytes larger than 2x2mm. Metacarpus 3 and Mt3 were dissected loose from the rest of the limb and the mid-medial and mid lateral condyles of Mc3 and Mt3, respectively, were identified using the transverse MRI template and sectioned into 3$5 \mathrm{~mm}$ thick slices using a band saw. The dorsal and medial aspects of each bone section were marked and the sample placed into an $8 \%$ nitric acid made up in $10 \%$ buffered formalin solution for fixation and decalcification. The solution was replaced every week to optimize demineralization until the bone floated in the solution. Sites 1, 2 and 3 were identified using the sagittal template of the mid-medial and mid- lateral distal condyles of Mc3 and Mt3, respectively, the cut blocks processed, embedded into paraffin wax, sectioned on a rotary microtome and stained using standard Haematoxylin and Eosin. Sections were then mounted with Entellan (Merck Chemicals, Darmstadt, Germany).

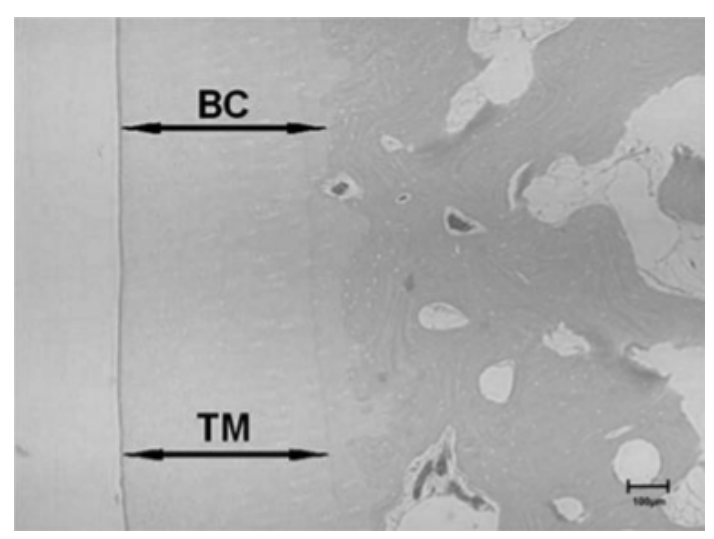

FIG. 2. Hematoxylin and Eosin histology slide indicating how measurements were made for the distance between the articular surface and the beginning of the Haversion canal system (BC, bone cartilage interface), and the distance between the articular surface and the beginning of the mineralized cartilage interface (TM, calcified cartilage tide mark).

For cartilage thickness measurement analysis, the stained sections were viewed with a Nikon (Centurion, South Africa) light microscope equipped with an 
Axio Cam camera (Axiovision VS40V4.8.1.0 Carl Zeiss Imaging Solutions GmbH). Two observers measured cartilage thickness from the articular surface to the first Haversian canal (bone cartilage interface) and from the articular surface to the mineralized cartilage interface (tide mark) if visible (Fig. 2). Three measurements were made at each site and the mean determined. Each set of metacarpo/metatarsophalangeal measurements was acquired at least 5 days apart.

To determine whether regions of interest to be mapped on the T2- and dGEMRICmapped images were representative of true cartilage dimensions, the histomorphometric thicknesses of cartilage at sites 1-3 were compared to measurements from MRI images ${ }^{28}$. Cartilage thickness was measured using a Siemens SYNGO workstation (Siemens Healthcare, Erlangen, Germany). The optimal inversion recovery sequence for visualizing the surface of the cartilage was determined subjectively by the first author and based on an evaluation of all 600 inversion recovery series of the dGEMRIC mapping images (4 limbs x 5 times (preGd, 30, 90, 120, 180 mins) x 5 sites (the extra 2 were not used in this study) x 6 different TIs $=600$ ) of a randomly picked horse's distal Mc3/Mt3 (Horse 6). Each image was zoomed and panned into an optimal position where a translucent template (Figure 1B) could determine the midline of the relevant condyle of $\mathrm{Mc} 3 / \mathrm{Mt} 3$ and the center of rotation of distal Mc3/Mt3. The optimal window was found using the windowing tool in the software package and the sites determined where the cartilage surface and bone cartilage interface could best be identified. The mineralized cartilage interface could not be visualized so no attempt was made to measure this. Based on results of these assessments, the $\mathrm{TI}=200 \mathrm{~ms}$ IR (STIR) in the 180 minute post Gd$\mathrm{DTPA}^{2-}$ time series was chosen as the best for measuring all horses' Mc3/Mt3 using the Syngo electronic callipers (Figure 3A). A similar procedure was used for the T2- 
mapping TEs (using all 24 limbs x 5 sites x $6 \mathrm{TE}=720$ sites) and the TE of $16.7 \mathrm{~ms}$ (PDw) was subjectively deemed the best image for visualizing the cartilage surface (Figure 3B). The thickness of the articular cartilage was then measured for sites 1-3 for all the PDw and STIR images of all 24 limbs, respectively, 3 times and the mean determined for each site and MRI measurement. This measurement procedure was repeated at least 2 months later by the same observer. Histologic cartilage thicknesses were evaluated at Sites 1-3 by the same observer at least 3 months after the MRI measurements.
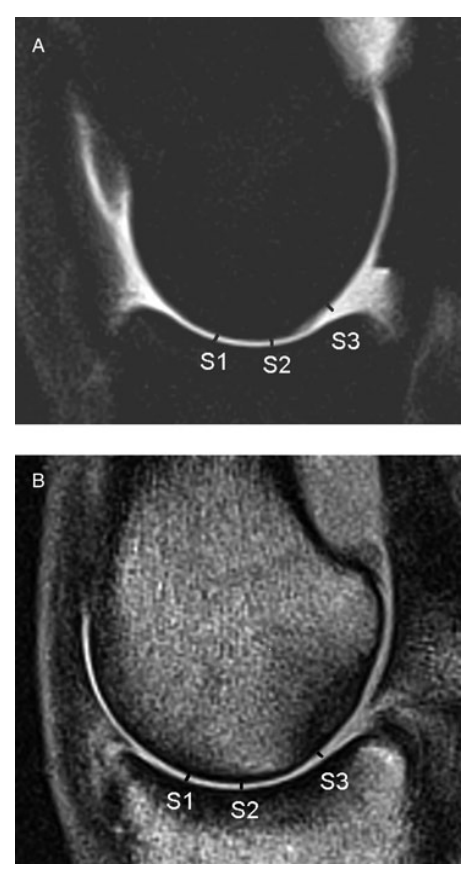

FIG. 3A. MRI STIR image at 180 minutes post Gd-DTPA ${ }^{2-}$ demonstrating a parasagittal slice of the distal mid-Mt3 condyle for Horse 5 and locations where the hyperintense articular cartilage depth was measured at sites 1-3. Window width 1734; window level 964.

FIG. 3B. Proton density weighted MRI image of the distal mid Mc3 condyle for Horse 4, demonstrating where the hyperintense articular cartilage depth was measured at sites 1-3. Window width 1445; window level 706. Note that since there is minimal synovial fluid within the joint and that this T2 image is prior to Gd injection. There is minimal fluid signal within the joint itself. $\mathrm{S}=$ site. 
Statistical analyses were performed using SPSS software (version 19, SPSS Inc, Chicago, IL, USA). The selection and conduction of all statistical tests were made in collaboration with statisticians. The level of significance for all tests was set at $\mathrm{p}<0.05$. Repeatability for cartilage thickness measurements was determined using the intraclass correlation coefficient (ICC), which represents the error-free proportion of the inter-subject score variation with a 95\% confidence interval (CI). The ICC values above 0.75 were classified as good, values between 0.74 and 0.40 as moderate and values below 0.40 were deemed to have poor reliability ${ }^{44}$ with a negative ICC also indicating low true intraclass correlation. ${ }^{45}$

Differences between cartilage thickness measurements were tested using Wilcoxon signed rank tests, performed post hoc. Forelimb and hindlimb cartilage thickness values $(\mathrm{mm})$ recorded from histologic bone cartilage interface, histologic mineralized cartilage interface, MRI STIR and MRI PDw were compared for sites 1, 2, and 3 separately as well as sites 1-3 combined using Wilcoxon signed rank tests.

\section{Results}

Twenty limbs met the inclusion criteria and four limbs were excluded, two for osteoarthritis, one for synovitis and the other for an osseous cyst-like lesion. T2mapping data was lost in Horse 1 right and left hind limbs, and Horse 2's right hind limb due to technical errors; therefore 17 limbs were available for T2-mapping ROI tracing.

Means, standard deviations and intra- and interobserver ICCs of cartilage thickness at sites 1-3 for bone cartilage interface and mineralized cartilage interface, and combined sites 1-3, respectively, are summarized in Table 1. For histomorphometric cartilage thickness using the bone cartilage interface, intra- 
TABLE 1. Mean and Standard Deviation values for Histological Cartilage Thickness of Distal Metacarpal 3 / Metatarsal 3 at Sites 1-3, Measured at Least 5 Days Apart by Observers A and B.

\begin{tabular}{|c|c|c|c|c|c|c|c|c|c|}
\hline & & & & & \multicolumn{5}{|c|}{$\mathrm{BC} \mathrm{mm}$ (SD) } \\
\hline \multirow[t]{2}{*}{ Site } & \multicolumn{4}{|c|}{ Observer A } & \multicolumn{5}{|c|}{ Observer B } \\
\hline & 1 & 2 & $\begin{array}{l}\mathrm{ICC} \\
1 \mathrm{vs} 2\end{array}$ & $\begin{array}{l}\text { Mean } \\
1 \& 2\end{array}$ & 1 & 2 & $\begin{array}{l}\text { ICC } \\
1 v s \\
2\end{array}$ & $\begin{array}{l}\text { Mean } \\
1 \& 2\end{array}$ & $\begin{array}{l}\text { ICC } \\
\text { BC means of } \\
A 1 \& 2 \text { vs } B 1 \& 2\end{array}$ \\
\hline 1 & $\begin{array}{l}0.87 \\
(0.11)\end{array}$ & $\begin{array}{l}0.88 \\
(0.10)\end{array}$ & 0.97 & $\begin{array}{l}0.87 \\
(0.10)\end{array}$ & $\begin{array}{l}0.88 \\
(0.10)\end{array}$ & $\begin{array}{l}0.86 \\
(0.07)\end{array}$ & 0.67 & $\begin{array}{l}0.87 \\
(0.07)\end{array}$ & 0.89 \\
\hline 2 & $\begin{array}{l}0.98 \\
(0.08)\end{array}$ & $\begin{array}{l}1.00 \\
(0.67)\end{array}$ & 0.80 & $\begin{array}{l}0.99 \\
(0.07)\end{array}$ & $\begin{array}{l}0.99 \\
(0.06)\end{array}$ & $\begin{array}{l}1.02 \\
(0.15)\end{array}$ & 0.61 & $\begin{array}{l}1.00 \\
(0.1)\end{array}$ & 0.66 \\
\hline 3 & $\begin{array}{l}0.79 \\
(0.14)\end{array}$ & $\begin{array}{l}0.77 \\
(0.10)\end{array}$ & 0.87 & $\begin{array}{l}0.79 \\
(0.11)\end{array}$ & $\begin{array}{l}0.77 \\
(0.08)\end{array}$ & $\begin{array}{l}0.74 \\
(0.12)\end{array}$ & 0.85 & $\begin{array}{l}0.76 \\
(0.09)\end{array}$ & 0.95 \\
\hline $1-3$ & $\begin{array}{l}0.88 \\
(0.08)\end{array}$ & $\begin{array}{l}0.89 \\
(0.06)\end{array}$ & 0.89 & $\begin{array}{l}0.88 \\
(0.07)\end{array}$ & $\begin{array}{l}0.88 \\
(0.05)\end{array}$ & $\begin{array}{l}0.87 \\
(0.07)\end{array}$ & 0.61 & $\begin{array}{l}0.88 \\
(0.05)\end{array}$ & 0.92 \\
\hline \multicolumn{10}{|c|}{ TM (SD) } \\
\hline Site & \multicolumn{4}{|c|}{ Observer A } & \multicolumn{5}{|c|}{ Observer B } \\
\hline & 1 & 2 & $\begin{array}{l}\text { ICC } \\
1 \text { vs } \\
2\end{array}$ & $\begin{array}{l}\text { Mean } \\
1 \& 2\end{array}$ & 1 & 2 & $\begin{array}{l}\text { ICC } \\
1 v s \\
2\end{array}$ & $\begin{array}{l}\text { Mean } \\
1 \& 2\end{array}$ & $\begin{array}{l}\text { ICC } \\
\text { means of } \\
\mathrm{A}(1 \& 2) \\
\text { vs } \mathrm{B}(1 \& 2)\end{array}$ \\
\hline 1 & $\begin{array}{l}0.64 \\
(0.1)\end{array}$ & $\begin{array}{l}0.63 \\
(0.09)\end{array}$ & 0.91 & $\begin{array}{l}0.64 \\
(0.09)\end{array}$ & $\begin{array}{l}0.62 \\
(0.10)\end{array}$ & $\begin{array}{l}0.60 \\
(0.08)\end{array}$ & 0.54 & $\begin{array}{l}0.61 \\
(0.07)\end{array}$ & 0.73 \\
\hline 2 & $\begin{array}{l}0.77 \\
(0.09)\end{array}$ & $\begin{array}{l}0.75 \\
(0.09)\end{array}$ & 0.66 & $\begin{array}{l}0.76 \\
(0.07)\end{array}$ & $\begin{array}{l}0.73 \\
(0.08)\end{array}$ & $\begin{array}{l}0.73 \\
(0.24)\end{array}$ & 0.55 & $\begin{array}{l}0.73 \\
(0.14)\end{array}$ & 0.66 \\
\hline 3 & $\begin{array}{l}0.62 \\
(0.12)\end{array}$ & $\begin{array}{l}0.59 \\
(0.06)\end{array}$ & 0.82 & $\begin{array}{l}0.61 \\
(0.09)\end{array}$ & $\begin{array}{l}0.59 \\
(0.05)\end{array}$ & $\begin{array}{l}0.54 \\
(0.11)\end{array}$ & 0.10 & $\begin{array}{l}0.57 \\
(0.06)\end{array}$ & 0.5 \\
\hline $1-3$ & $\begin{array}{l}0.67 \\
(0.07)\end{array}$ & $\begin{array}{l}0.66 \\
(0.05)\end{array}$ & 0.76 & $\begin{array}{l}0.67 \\
(0.05)\end{array}$ & $\begin{array}{l}0.64 \\
(0.05)\end{array}$ & $\begin{array}{l}0.63 \\
(0.08)\end{array}$ & -0.21 & $\begin{array}{l}0.63 \\
(0.04)\end{array}$ & 0.37 \\
\hline
\end{tabular}

$\mathrm{BC}$ : measurement from the articular surface to the beginning of the Haversian canals; TM:

measurement from the articular surface to the beginning of the mineralised cartilage (tide mark); ICC = intraclass correlation coefficient; $\mathrm{SD}=$ standard deviation, $\mathrm{mm}=$ millimeters, $\mathrm{Mc} 3=$ metacarpal 3, Mt3 $=$ metatarsal 3 .

observer repeatability for Observer A was good, with the least repeatable results at site 2 (ICC 0.80$)$ and the most repeatable results at site $1(0.97)$. Intra-observer repeatability for Observer B was good to moderate, with the most repeatable results at site $3(0.85)$ and less repeatable results for site $2(0.61)$. Repeatability based on means of Observer A1 and Observer A2 measurements and means of Observer B1 and Observer B2 measurements was good $(\geq 0.89)$ for sites 1 and 3 , and moderate $(0.66)$ for site 2 . 
For histomorphometric cartilage thickness using the mineralized cartilage interface, intra-observer repeatability for Observer A was good at sites 1 and 3, with least repeatability for site $2(0.66)$ and most repeatability for site $1(0.91)$. Observer B measurements were moderately repeatable for sites 1 and 2, but poorly repeatable for site $3(0.10)$. The repeatability between means of Observer A1 and Observer A2 measurements, versus means of Observer B1 and Observer B2 measurements, was moderate for sites 1,2 and 3, respectively.

TABLE 2. Mean and Standard Deviation Values for Cartilage Thickness of Distal Metacarpal 3 / Metatarsal 3, at Sites 1-3, Measured using a STIR Sequence at 180 minutes post Gd-DTPA ${ }^{2-}$ and a Proton Density weighted T2 Mapping Sequence, Repeated at Least 5 days Apart by Observer A.

\begin{tabular}{lllllllll}
\hline Site & \multicolumn{3}{c}{$\mathrm{STIR}(\mathrm{SD}) \mathrm{mm}$} & \multicolumn{5}{c}{ PDw (SD) mm } \\
\hline & 1 & 2 & $\begin{array}{l}\text { ICC } \\
1 \text { vs }\end{array}$ & $\begin{array}{l}\text { mean } \\
1 \& 2\end{array}$ & 1 & 2 & $\begin{array}{l}\text { ICC } \\
1 \text { vs 2 }\end{array}$ & $\begin{array}{l}\text { mean } \\
1 \& 2\end{array}$ \\
\hline 1 & 0.56 & 0.53 & 0.11 & 0.54 & 0.54 & 0.56 & 0.77 & 0.55 \\
& $(0.06)$ & $(0.02)$ & & $(0.04)$ & $(0.08)$ & $(0.07)$ & & $(0.07)$ \\
2 & 0.51 & 0.54 & 0.58 & 0.52 & 0.44 & 0.51 & 0.31 & 0.47 \\
& $(0.07)$ & $(0.07)$ & & $(0.06)$ & $(0.05)$ & $(0.06)$ & & $(0.05)$ \\
3 & 0.87 & 0.80 & 0.79 & 0.84 & 0.77 & 0.86 & 0.69 & 0.81 \\
& $(0.13)$ & $(0.15)$ & & $(0.13)$ & $(0.1)$ & $(0.13)$ & & $(0.11)$ \\
4 & 1.02 & 1.03 & 0.73 & 1.03 & 0.95 & 0.93 & -0.15 & 0.94 \\
& $(0.06)$ & $(0.08)$ & & $(0.06)$ & $(0.1)$ & $(0.06)$ & & $(0.05)$ \\
5 & 0.94 & 0.91 & 0.74 & 0.92 & 0.83 & 0.85 & 0.91 & 0.84 \\
& $(0.06)$ & $(0.05)$ & & $(0.05)$ & $(0.06)$ & $(0.08)$ & & $(0.07)$ \\
$1-3$ & 0.65 & 0.62 & 0.48 & 0.63 & 0.71 & 0.74 & 0.47 & 0.72 \\
& $(0.07)$ & $(0.07)$ & & $(0.06)$ & $(0.05)$ & $(0.05)$ & & $(0.04)$ \\
$1-5$ & 0.78 & 0.76 & 0.70 & 0.77 & 0.58 & 0.64 & 0.53 & 0.61 \\
& $(0.07)$ & $(0.05)$ & & $(0.05)$ & $(0.05)$ & $(0.05)$ & & $(0.04)$ \\
\hline
\end{tabular}

$\mathrm{STIR}=$ short tau inversion recovery; $\mathrm{PDw}=$ proton density weighted; ICC $=$ intraclass correlation coefficient; $\mathrm{SD}=$ standard deviation, $\mathrm{mm}=$ millimeters, $\mathrm{Mc} 3=$ metacarpal $3, \mathrm{Mt} 3=$ metatarsal 3

Means, standard deviations and intra- observer ICCs of cartilages thicknesses at sites 1-3, separately, and 1-3 combined, for the STIR images at 180 minutes post Gd-DTPA $^{2-}$ and the PDw images are summarized in Table 2. For the STIR cartilage thickness measurements, Observer A showed good repeatability for site 3 (0.79), moderate repeatability for site $2(0.58)$ and poor repeatability for Site $1(0.11)$. For the 
PDw cartilage thickness measurements, Observer A showed good repeatability for site $1(0.77)$, moderate repeatability for site $3(0.69)$ and poor repeatability for site 2 $(0.31)$

TABLE 3. Mean, Standard Deviation values and Wilcoxon Signed Rank Evaluation of Mean Measurements of Cartilage Thickness of Distal Metacarpal 3 / Metatarsal 3 Measured by Observer A, at Sites 1-3 using MRI and Histologic Techniques.

\begin{tabular}{|c|c|c|c|c|c|c|}
\hline Site & Histomorpho & netric (SD) & STIR (SD) & $\begin{array}{l}\text { PDw } \\
\text { (SD) }\end{array}$ & Wilcoxon signe & test \\
\hline & $\begin{array}{l}\mathrm{BC} \\
\mathrm{mm}\end{array}$ & $\begin{array}{l}\text { TM } \\
\mathrm{mm}\end{array}$ & $\mathrm{mm}$ & $\mathrm{mm}$ & $\begin{array}{l}\text { Technique } \\
\text { comparison }\end{array}$ & $p$ \\
\hline 1 & $0.87(0.10)$ & $0.64(0.09)$ & $0.54(0.04)$ & $\begin{array}{l}0.55 \\
(0.07)\end{array}$ & $\begin{array}{l}\text { BC vs STIR } \\
\text { BC vs PDw } \\
\text { TM vs STIR } \\
\text { TM vs PDw } \\
\text { PDw vs STIR }\end{array}$ & $\begin{array}{l}0.031^{*} \\
0.031^{*} \\
0.063 \\
0.063 \\
0.563\end{array}$ \\
\hline 2 & $0.99(0.07)$ & $0.76(0.07)$ & $0.52(0.06)$ & $\begin{array}{l}0.47 \\
(0.05)\end{array}$ & $\begin{array}{l}\text { BC vs STIR } \\
\text { BC vs PDw } \\
\text { TM vs STIR } \\
\text { TM vs PDw } \\
\text { PDw vs STIR }\end{array}$ & $\begin{array}{l}0.031^{*} \\
0.031^{*} \\
0.031^{*} \\
0.031^{*} \\
0.688\end{array}$ \\
\hline 3 & $0.79(0.11)$ & $0.61(0.09)$ & $0.84(0.13)$ & $\begin{array}{l}0.81 \\
(0.11)\end{array}$ & $\begin{array}{l}\text { BC vs STIR } \\
\text { BC vs PDw } \\
\text { TM vs STIR } \\
\text { TM vs PDw } \\
\text { PDw vs STIR }\end{array}$ & $\begin{array}{l}0.69 \\
0.563 \\
0.063 \\
0.031^{*} \\
0.688\end{array}$ \\
\hline 4 & - & - & $1.030 .06)$ & $\begin{array}{l}0.94 \\
(0.05)\end{array}$ & STIR vs PDw & 0.156 \\
\hline 5 & - & - & $0.92(0.05)$ & $\begin{array}{l}0.84 \\
(0.07)\end{array}$ & STIR vs PDw & $0.031^{*}$ \\
\hline $1-3$ & $0.88(0.07)$ & $0.67(0.05)$ & $0.63(0.06)$ & $\begin{array}{l}0.61 \\
(0.04)\end{array}$ & $\begin{array}{l}\text { BC vs STIR } \\
\text { BC vs PDw } \\
\text { TM vs STIR } \\
\text { TM vs PDw } \\
\text { PDw vs STIR }\end{array}$ & $\begin{array}{l}0.031^{*} \\
0.031^{*} \\
0.688 \\
0.031^{*} \\
0.563\end{array}$ \\
\hline $1-5$ & - & - & $0.77(0.05)$ & $\begin{array}{l}0.72 \\
(0.04)\end{array}$ & PDw vs STIR & 0.156 \\
\hline
\end{tabular}

STIR (short tau inversion recovery) $=I R$ measured on $T I=200 \mathrm{msec}^{\text {at }} 180$ minutes post Gd-DTPA ${ }^{2-}$ sequence; PDw (proton density weighted) = a T2-mapping sequence at $\mathrm{TE}=16.7 \mathrm{~ms}$; Wilcoxon signed rank test (2-tailed). Significant differences at $\mathrm{P}<0.05$ are marked with*. $\mathrm{BC}=$ articular surface to bone-cartilage interface; $\mathrm{TM}=$ articular surface to beginning of mineralised cartilage; $\mathrm{SD}=$ standard deviation, $\mathrm{mm}=$ millimeters, $\mathrm{Mc} 3=$ metacarpal $3, \mathrm{Mt} 3=$ metatarsal 3 
Table 3 summarizes the Wilcoxon signed rank test results for cartilage thickness differences among histologic bone cartilage interface, histologic mineralized cartilage interface, MRI STIR and MRI PDw at sites 1-3 (separately and combined); and between MRI STIR and MRI PDw images at sites 1-3 (separately and combined). Histologic cartilage thickness measured using the bone cartilage interface differed significantly from MRI STIR and MRI PDw measurements at sites 1 and 2 and at sites 1-3 combined, but not at site $3(\mathrm{p}<0.05)$. Histologic cartilage thickness measured using the mineralized cartilage interface differed significantly from MRI STIR and MRI PDw measurements at site 2 and differed from MRI PDw at site 3 and sites 1-3 combined $(\mathrm{p}<0.05)$. Histologic cartilage thickness measured using the mineralized cartilage interface differed moderately from MRI STIR cartilage thickness at sites 1 and $3(p=0.063){ }^{46}$ Cartilage thickness from MRI PDw did not significantly differ from MRI STIR cartilage thickness at any sites. No differences were found between forelimb and hindlimb cartilage thickness values for all measurement methods; sites 1, 2, and 3 separately; and sites 1-3 combined using Wilcoxon signed rank tests and $\mathrm{p}<0.05$.

\section{Discussion}

The most important finding in this study was that the histological bone interface cartilage thickness and the STIR and PDw cartilage thicknesses of Mc3/Mt3 did not differ significantly when the distal Mc3/Mt3 was not in contact with the proximal phalanx, validating the STIR and PDw measurements for later studies using these sequences and particularly dGEMRIC and T2 mapping of articular cartilage of the distal Mc3/Mt3. It has previously been reported that cartilage thickness for dorsal and palmar sites of distal Mc3 in racehorses is approximately $0.79 \pm 0.16 \mathrm{~mm}$ when measured histologically, and $0.90 \pm 0.17 \mathrm{~mm}$ when measured using fat-suppressed 
spoiled gradient-recalled images. ${ }^{47}$ The current study's mean histological cartilage thickness measurement was slightly higher, which could be a result of individual observer technique, e.g. Observer A and B's measurements did differ significantly at some sites. Overestimation of cartilage thickness when the actual thickness is $<1 \mathrm{~mm}$ thick has been observed with MRI-based 3D cartilage models. ${ }^{48}$ This is similar to findings in the current study where the STIR and PDw bone cartilage interface measurements were larger than the histomorphometric dimensions at site 3 where the cartilage surface could be seen and less at sites 1 and 2 where the cartilage made contact with phalanx one. For sites 1 and 2, when windowing the STIR and PDw images to attempt to identify the articular cartilage surface, partial volume averaging of the thin intra-articular space (often on the 1 pixel threshold) tended to determine the boundary between the articular surfaces of distal Mc3/Mt3 and proximal phalanx to be half way between them, resulting in a halving of the measured distance. In a previous human knee cartilage report, the weight-bearing and central areas on each femoral and tibial condyle yielded more accurate measurements than boundary and non-weight-bearing regions based on sagittal plane MR imaging. ${ }^{8}$ However the normal human knee cartilage is 1.3 to $2.5 \mathrm{~mm}$ thick versus the approximately $1 \mathrm{~mm}$ thickness of the distal Mc3/Mt3 horse cartilage. Human knee cartilage also has a meniscus which is not present in the equine metacarpo/metatarsophalangeal joint, therefore similar comparisons with the equine $\mathrm{Mc} 3 / \mathrm{Mt} 3$ joint cannot be made. ${ }^{49}$ The use of traction to separate the two cartilage surfaces and better evaluate them has been described in human knees ${ }^{50}$ and should therefore be considered in the future when evaluating the metacarpo/metatarsophalangeal cartilages using dGEMRIC and T2 mapping in the horse and in the clinical setting. 
Histomorphometrically, the bone cartilage interface measurements of cartilage thickness were consistently higher than the mineralized cartilage interface measurements. This finding was expected, since the tideline of calcified cartilage is found in the articular cartilage between the Haversian canals and the cartilage surface. The amount of mineralized cartilage interface measurements that could be made with confidence were less than the bone cartilage interface measurements, because the border of the mineralised cartilage could not be clearly ascertained in several of the slides.

Mineralized cartilage interface cartilage thicknesses were consistently found to be higher than STIR and PDw measurements at sites 1 and 2, but lower at site 3, likely for the same reason as for the bone cartilage interface measurements. These findings support previously reported findings where MRI measurements of equine carpal cartilage were significantly greater than the histologic measurements. ${ }^{28}$

The subjective choice of STIR and PDw sequences as being the best for visualizing cartilage in the current study was supported by the fact that $\mathrm{TI}=200 \mathrm{~ms}$ is quite close to STIR imaging at $1.5 \mathrm{~T}$ (where $\mathrm{TI}=150 \mathrm{~ms}$ ), which means that the fat signal of the image is close to zero, and cartilage with relatively high water content can be well differentiated from its surroundings. For T2, the image with the shortest TE has the best signal to noise ratio and least $\mathrm{T} 2$ weighting, therefore closer to a proton density weighting, which yields a good contrast between cartilage and surrounding tissues. One possible limitation of the study was that only one horse's limb was used to determine the selection of these sequences. However, a very rigorous review process was followed for all the limbs to ensure the cartilage examined was as normal as possible. Also, the MRI properties of fat and water were 
expected to be constant and behave similarly when using a constant set of imaging parameters at a constant field strength.

It was encouraging that findings from the current study indicated that intraobserver repeatability of MRI measurements was good to moderate where cartilage was not in contact with other cartilage. This finding was similar to previously published findings. ${ }^{29-31}$ One limitation of the current study was that no inter-observer comparisons were made. However, previous studies have found good inter-observer repeatability for MRI measurements.

Another limitation of the current study was that histomorphometric measurements were not measured at more sites away from adjacent cartilage. Histomorphometric cartilage thickness measurements of the dorsal and palmar aspects of distal Mc3 have not been found to differ in previous studies. ${ }^{47}$ Therefore, it is reasonable to assume that measurements from these sites would be relatively similar to site 3's measurements.

If a higher field strength magnet had been used, higher resolution images could have resulted in superior cartilage thickness measurements as has been reported in a human study comparing $3 \mathrm{~T}$ with $1.5 \mathrm{~T}$ images. However, even in that study, correlation coefficients for values obtained at $3 \mathrm{~T}$ and $1.5 \mathrm{~T}$ were high. ${ }^{51}$ Using thinner slices for MRI scans was considered for the current study, but this would have resulted in a lower signal to noise ratio and would have been more time-consuming. The effect of slice thickness on assessment of human knee cartilage volume has been previously reported and findings indicated that there was little difference in human tibial cartilage volume as slice thickness increased from 1.5 to $7.5 \mathrm{~mm} .{ }^{52}$ Conclusions from the previous study were that increasing slice thicknesses could be used and that this would result in decreased acquisition and post-processing times. However, since 
human knee cartilage thickness is much greater than that of the equine fetlock, this extrapolation may not be valid in equine fetlock joints. Cartilage curvature is another important factor to consider. If cartilage is very thin but not curved in the region being evaluated, thicker slices will also give reliable results. The small sites evaluated in the distal Mc3/Mt3 had very little curvature. Another limitation to this study was the relatively low number of horses and limbs used, decreasing the power of the findings. In spite of these limitations, bone cartilage interface histomorphometric cartilage thickness measurements did not differ from MRI measurements using a selected inversion recovery sequence for dGEMRIC mapping, and a selected time to echo image for T2 mapping in the palmaro/plantarodistal aspect of the distal Mc3/Mt3. This finding validates the use of dGEMRIC and T2 mapping for measuring cartilage thickness in locations where cartilage is not in close approximation to opposing adjacent cartilage in Mc3/Mt3 of Thoroughbred horses. Future studies are needed to evaluate these dGEMRIC and T2 mapping techniques in live horses with and without joint disease. 


\section{REFERENCES}

1. Dyson PK, Jackson BF, Pfeiffer DU, Price JS. Days lost from training by two- and three-year-old Thoroughbred horses: A survey of seven UK training yards. Equine Vet J 2008;40:650-7.

2. Perkins NR, Reid SW, Morris RS. Profiling the New Zealand Thoroughbred racing industry. 2. conditions interfering with training and racing. N Z Vet J 2005;53:69-76.

3. Parente EJ, Russau AL, Birks EK. Effects of mild forelimb lameness on exercise performance. Equine Vet J Suppl 2002; 34:252-6.

4. Hernandez J, Hawkins DL. Training failure among yearling horses. Am J Vet Res 2001;62:1418-22.

5. Bailey CJ, Reid SW, Hodgson DR, Bourke JM, Rose RJ. Flat, hurdle and steeple racing: Risk factors for musculoskeletal injury. Equine Vet J 1998;30:498-503.

6. Olivier A, Nurton JP, Guthrie AJ. An epizoological study of wastage in Thoroughbred racehorses in Gauteng, South Africa J S Afr Vet Assoc 1997;68:125-9.

7. A comparison of the economic costs of equine lameness, colic, and equine protozoal myeloencephalitis (EPM) in the United States. Animal and Plant Health Inspection service. October 2001; \#N348.1001 (available at http://www.aphis.usda.gov/animal_health/nahms/equine/downloads/equine98/Equine 98 is EconCost.pdf (accessed May 2011). 
8. Broster CE, Burn CC, Barr AR, Whay HR. The range and prevalence of pathological abnormalities associated with lameness in working horses from developing countries. Equine Vet J 2009;41:474-81.

9. de Grauw JC, van de Lest CH, van Weeren R, Brommer H, Brama PA. Arthrogenic lameness of the fetlock: Synovial fluid markers of inflammation and cartilage turnover in relation to clinical joint pain. Equine Vet J 2006;38:305-11.

10. Wilsher S, Allen WR, Wood JL. Factors associated with failure of thoroughbred horses to train and race. Equine Vet J 2006;38:113-8.

11. Dabareiner RM, Cohen ND, Carter GK, Nunn S, Moyer W. Musculoskeletal problems associated with lameness and poor performance among horses used for barrel racing: 118 cases (2000-2003). J Am Vet Med Assoc 2005;227:1646-50.

12. Rossdale PD, Hopes R, Digby NJ, Offord K. Epidemiological study of wastage among racehorses 1982 and 1983. Vet Rec 1985;116:66-9.

13. Bailey CJ, Reid SW, Hodgson DR, Rose RJ. Impact of injuries and disease on a cohort of two- and three-year-old Thoroughbreds in training. Vet Rec 1999;145:48793.

14. Brommer H, van Weeren PR, Brama PA, Barneveld A. Quantification and agerelated distribution of articular cartilage degeneration in the equine fetlock joint. Equine Vet J 2003;35:697-201.

15. Pool RR. Pathologic manifestations of joint disease in the athletic horse. In: McIlwraith CW, Trotter GW (eds): Joint disease in the horse. Philadelphia: W.B.Saunders Co. 1996;87-104. 
16. McIlwraith CW. General pathology of the joint and response to injury. In:

McIlwraith CW, Trotter GW (eds): Joint disease in the horse. Philadelphia: WB Saunders Co. 2006;40-70.

17. Martel-Pelletier J. Pathophysiology of osteoarthritis. Osteoarthritis Cartilage 2004;12 Suppl A:S31-3.

18. Pearle AD, Warren RF, Rodeo SA. Basic science of articular cartilage and osteoarthritis. Clin Sports Med 2005;24:1-12.

19. Rannou F, Sellam J, Berenbaum F. Pathophysiology of osteoarthritis: Updated concepts. Presse Med 2010;26.

20. Goldring MB, Goldring SR. Osteoarthritis. J Cell Physiol 2007;213:626-34.

21. Gold GE, Burstein D, Dardzinski B, Lang P, Boada F, Mosher T. MRI of articular cartilage in OA: Novel pulse sequences and compositional/functional markers. Osteoarthritis Cartilage 2006;14 Suppl A:A76-86.

22. Guermazi A, Burstein D, Conaghan P, Eckstein F, Graverand HL, Keen H, et al. Imaging in osteoarthritis. Rheum Dis Clin North Am 2008;34:645-87.

23. Trattnig S, Domayer S, Welsch GW, Mosher T, Eckstein F. MR imaging of cartilage and its repair in the knee -a review. Eur Radiol 2009;19:1582-94.

24. Roemer FW, Eckstein F, Guermazi A. Magnetic resonance imaging-based semiquantitative and quantitative assessment in osteoarthritis. Rheum Dis Clin North Am 2009;35:521-55. 
25. Potter HG, Black BR, Chong le R. New techniques in articular cartilage imaging. Clin Sports Med 2009;28:77-94.

26. Domayer SE, Welsch GH, Dorotka R, Mamisch TC, Marlovits S, Szomolanyi P, et al. MRI monitoring of cartilage repair in the knee: A review. Semin Musculoskelet Radiol 2008;12:302-17.

27. Goodwin DW. Visualization of the macroscopic structure of hyaline cartilage with MR imaging. Semin Musculoskelet Radiol 2001;5:305-12.

28. Murray RC, Branch MV, Tranquille C, Woods S. Validation of magnetic resonance imaging for measurement of equine articular cartilage and subchondral bone thickness. Am J Vet Res 2005;66:1999-2005.

29. Zuo J, Bolbos R, Hammond K, Li X, Majumdar S. Reproducibility of the quantitative assessment of cartilage morphology and trabecular bone structure with magnetic resonance imaging at 7 T. Magn Reson Imaging 2008;26:560-6.

30. Naish JH, Xanthopoulos E, Hutchinson CE, Waterton JC, Taylor CJ. MR measurement of articular cartilage thickness distribution in the hip. Osteoarthritis Cartilage 2006;14:967-73.

31. McGibbon CA. Inter-rater and intra-rater reliability of subchondral bone and cartilage thickness measurement from MRI. Magn Reson Imaging 2003;21:707-14.

32. Graichen H, von Eisenhart-Rothe R, Vogl T, Englmeier KH, Eckstein F.

Quantitative assessment of cartilage status in osteoarthritis by quantitative magnetic resonance imaging: Technical validation for use in analysis of cartilage volume and further morphologic parameters. Arthritis Rheum 2004;50:811-6. 
33. Graichen H, Jakob J, von Eisenhart-Rothe R, Englmeier KH, Reiser M, Eckstein F. Validation of cartilage volume and thickness measurements in the human shoulder with quantitative magnetic resonance imaging. Osteoarthritis Cartilage 2003;11:47582.

34. Raynauld JP, Martel-Pelletier J, Abram F, Dorais M, Haraoui B, Choquette D, et al. Analysis of the precision and sensitivity to change of different approaches to assess cartilage loss by quantitative MRI in a longitudinal multicenter clinical trial in patients with knee osteoarthritis. Arthritis Res Ther 2008;10:R129.

35. Bolbos R, Benoit-Cattin H, Langlois JB, Chomel A, Chereul E, Odet C, et al. Measurement of knee cartilage thickness using MRI: A reproducibility study in a meniscectomized guinea pig model of osteoarthritis. NMR Biomed 2008;21:366-75.

36. Bolbos R, Benoit-Cattin H, Langlois JB, Chomel A, Chereul E, Odet C, et al. Knee cartilage thickness measurements using MRI: A 4(1/2)-month longitudinal study in the meniscectomized guinea pig model of OA. Osteoarthritis Cartilage 2007;15:656-65.

37. Kauffmann C, Gravel P, Godbout B, Gravel A, Beaudoin G, Raynauld JP, et al. Computer-aided method for quantification of cartilage thickness and volume changes using MRI: Validation study using a synthetic model. IEEE Trans Biomed Eng 2003;50:978-88.

38. Brem MH, Lang PK, Neumann G, Schlechtweg PM, Schneider E, Jackson R, et al. Magnetic resonance image segmentation using semi-automated software for quantification of knee articular cartilage-initial evaluation of a technique for paired scans. Skeletal Radiol 2009;38:505-11. 
39. Koo S, Giori NJ, Gold GE, Dyrby CO, Andriacchi TP. Accuracy of 3D cartilage models generated from MR images is dependent on cartilage thickness: Laser scanner based validation of in vivo cartilage. J Biomech Eng 2009;131:121004.

40. Hannila I, Nieminen MT, Rauvala E, Tervonen O, Ojala R. Patellar cartilage lesions: Comparison of magnetic resonance imaging and $\mathrm{T} 2$ relaxation-time mapping. Acta Radiol 2007;48:444-8.

41. Multanen J, Rauvala E, Lammentausta E, Ojala R, Kiviranta I, Hakkinen A, et al. Reproducibility of imaging human knee cartilage by delayed gadolinium-enhanced MRI of cartilage (dGEMRIC) at 1.5 Tesla. Osteoarthritis Cartilage 2009;17:559-64.

42. Firth EC, Doube M, Boyde A. Changes in mineralised tissue at the site of origin of condylar fracture are present before athletic training in Thoroughbred horses. NZ Vet J 2009;57:278-283.

43. O'Brien T, Baker TA, Brounts SH, Sample SJ, Markel MD, Scollay MC, et al. Detection of articular pathology of the distal aspect of the third metacarpal bone in Thoroughbred racehorses: comparison of radiography, computed tomography and magnetic resonance imaging. Vet Surg 2011;40:942-951.

44. Bland JM, Altman DG. Measurement error and correlation coefficients. BMJ $1996 ; 313: 41-2$.

45. Taylor PJ. An introduction to intraclass correlation that resolves some common confusions. 2011 Available at: http://faculty.umb.edu/pjt/9b.pdf. Accessed 21 July 2011. 
46. Albright SC, Winston WL, Zappe CJ. Data analysis and decision making. Australia: South-Western Cengage Learning. 2010;503.

47. Olive J, D'Anjou MA, Girard C, Laverty S, Theoret C. Fat-suppressed spoiled gradient-recalled imaging of equine metacarpophalangeal articular cartilage. Vet Radiol Ultrasound 2010;51:107-15.

48. Koo S, Gold GE, Andriacchi TP. Considerations in measuring cartilage thickness using MRI: Factors influencing reproducibility and accuracy. Osteoarthritis Cartilage 2005; 13:782-9.

49. Eckstein F, Reiser M, Englmeier KH, Putz R. In vivo morphometry and functional analysis of human articular cartilage with quantitative magnetic resonance imagingfrom image to data, from data to theory. Anat Embryol 2001;203:147-173

50. Nakanishi K, Tanaka H, Nishii T, Masuhara K, Narumi Y, Nakamura H. MR evaluation of the articular cartilage of the femoral head during traction. Correlation with resected femoral head. Acta Radiol 1999;40:60-3.

51. Eckstein F, Charles HC, Buck RJ, Kraus VB, Remmers AE, Hudelmaier M, et al. Accuracy and precision of quantitative assessment of cartilage morphology by magnetic resonance imaging at 3.0T. Arthritis Rheum 2005;52:3132-6.

52. Cicuttini F, Morris KF, Glisson M, Wluka AE. Slice thickness in the assessment of medial and lateral tibial cartilage volume and accuracy for the measurement of change in a longitudinal study. J Rheumatol 2004;31:2444-8. 